\title{
An optical telemetry device to identify which dolphin produces
} a sound

\author{
Peter Tyack \\ Department of Biology, Woods Hole Oceanographic Institution, Woods Hole, Massachusetts 02543
}

(Received 13 May 1985; accepted for publication 19 June 1985)

\begin{abstract}
A small telemetry device, called a "vocalight," was designed for attachment to a dolphin's head using a suction cup. The vocalight lights up a variable number of light-emitting diodes depending upon the loudness of sounds received at a hydrophone within the suction cup. If vocalights matched for sensitivity are put on each dolphin within a captive group, observers can identify which dolphin produces a vocalization. Use of vocalights indicates that source levels of whistles from captive bottlenosed dolphins, Tursiops truncatus, range from approximately 125 to over 140 $\mathrm{dB}$ re: $1 \mu \mathrm{Pa}$ at $1 \mathrm{~m}$.
\end{abstract}

PACS numbers: 43.80.Ev, 43.80.Nd

\section{INTRODUCTION}

A primary obstacle to progress in the study of dolphin communication has been the inability of researchers to determine which animal produces a vocalization within a socially interacting group. Dolphins occasionally emit a stream of bubbles while vocalizing, or make sounds audible in air. When observers can see the bubble stream or hear and localize a dolphin sound in air, they can often identify the source. But these events do not occur frequently enough to allow systematic analysis.

Several investigators have attempted to study communication between isolated captive dolphins using an electronic acoustic link between two pools (Lang and Smith, 1965; Burdin et al., 1975; Gish, 1979). But this approach does not completely solve the problem, for the isolated dolphins are able to interact only acoustically, and the electronic reproduction of dolphin sounds may be discriminably different from natural sounds.

The need for a technique to determine which animal produces which sound during normal social interaction has been discussed for over two decades. One obvious solution to the problem is to use a telemetry device to transmit information about sound production from each dolphin in the pool. Evans and Sutherland (1963) proposed the development of a 27-MHz radio telemetry device to broadcast sounds from a dolphin's head. This technique is limited to applications where the antenna remains above water, for the conductivity of seawater limits electromagnetic transmission, with losses increasing with frequency (Mackay, 1968).

The use of sound for underwater telemetry is common, since sound has favorable propagation characteristics underwater. But there are serious limitations to the use of sonic telemetry with dolphins. If the sounds of the telemetry device are audible to the animal, they may interfere with normal behavior. Many species of dolphin have hearing thresholds as high as $150-200 \mathrm{kHz}$ (Popper, 1980). Frequencies higher that this are difficult to work with for sonic telemetry.
This paper reports the development of a telemetry device, called a vocalight, that uses light emitting diodes (LEDs) to broadcast the level of sounds produced by a dolphin. The vocalight has been successfully used to study the whistle repertoires of two captive bottlenosed dolphins, Tursiops truncatus (Tyack, in press).

\section{DESCRIPTION OF THE VOCALIGHT}

A circuit diagram of the vocalight is presented in Fig. 1. A small piezoelectric ceramic disk (Channel Industries type 5500 , standard disk $1.27 \mathrm{~cm}$ in diameter by $0.32 \mathrm{~cm}$ thick) is used as a contact hydrophone to pick up the sounds of a dolphin. This signal is ac coupled to a two-stage amplifier. The feedback resistor in the first stage is a potentiometer allowing an adjustable gain of 20-60 dB. Small capacitors are added in parallel to the feedback resistors to improve stability. The amplification stage also incorporates a twopole, high-pass filter with a 3-dB point at $2 \mathrm{kHz}$. The highpass filter is designed to reduce low-frequency noise both from water pumps and from water flow along the device, while not reducing sensitivity to dolphin whistle vocalizations. The 2-kHz cutoff point is a compromise between these goals. While the device does respond to high-frequency turbulence as a dolphin breaks the water surface, it is not triggered by flow noise underwater, even during high-speed maneuvering.

The amplified and filtered signal is input to a LED display driver integrated circuit (LM 3915), which compares the input signal to a $1.2-\mathrm{V}$ reference voltage. The dynamic range of the circuit is $30 \mathrm{~dB}$. If the input signal is $30 \mathrm{~dB}$ less than the reference voltage, no LEDs light up. For every doubling of signal ( $3 \mathrm{~dB})$ above this level, another LED is turned on. If the signal exceeds the reference voltage, the device is saturated, and all ten LEDs remain turned on.

The dimensions of the vocalight are indicated in Fig. 2. The circuit is constructed on the battery case, which is a fiberglass tube, with the light emitting diodes oriented at the 


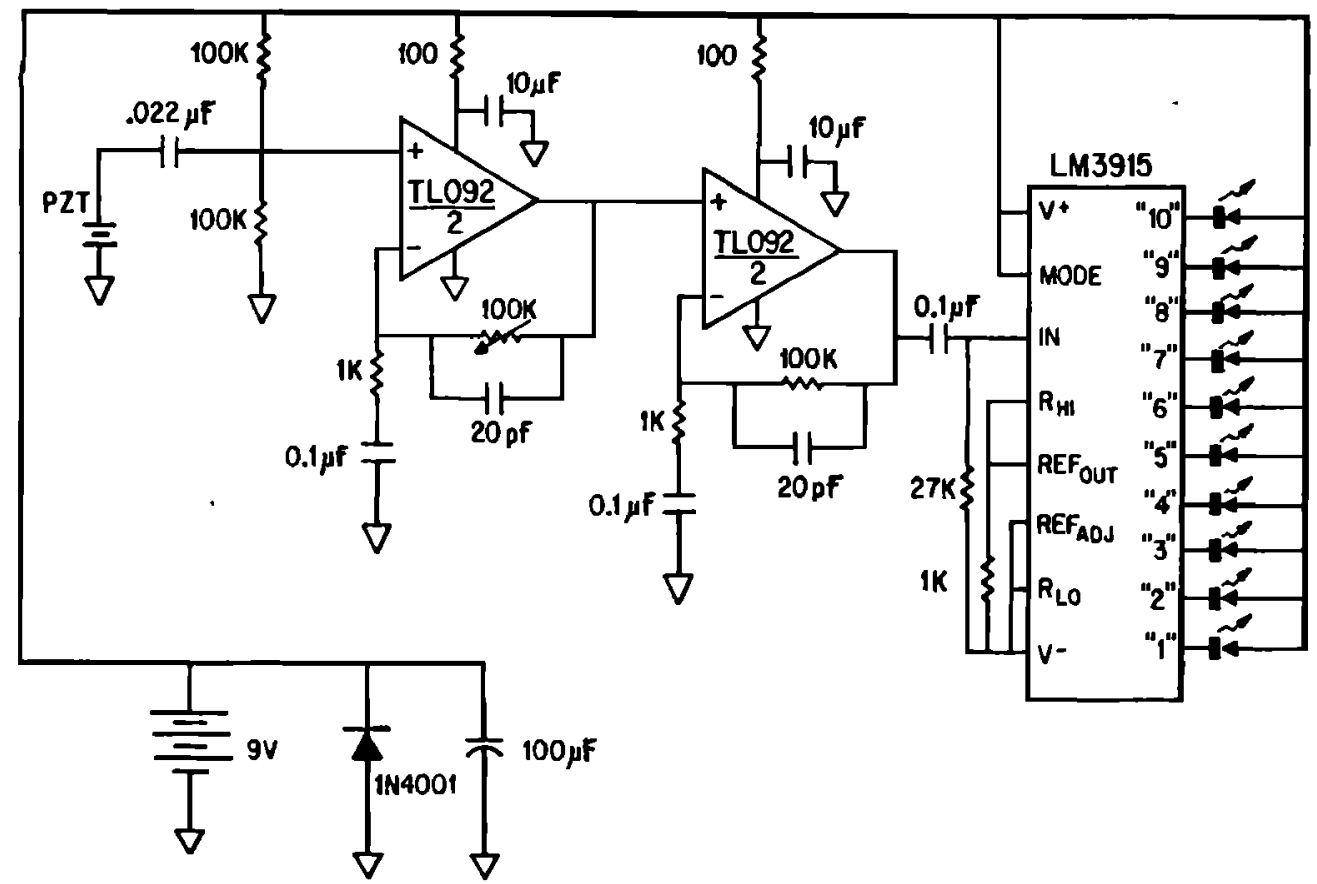

FIG. 1. Schematic diagram of vocalight circuit.

top of the tube and the contact hydrophone at the bottom. Once the circuit is constructed and tested, it is potted in epoxy (Hysol RE 2039 resin and HD 3469 hardener) using an epoxy mold. The circuit is then attached to a suction cup (Atlantic India suction cup type 472, $5.08 \mathrm{~cm}$ in diameter, made from compound 13312) using the same epoxy. In this step, the hydrophone element is sealed with a thin film of epoxy at the base of the suction cup. The contact hydrophone is backed by closed cell foam to reduce the strength of signals coming from any direction other than the surface on
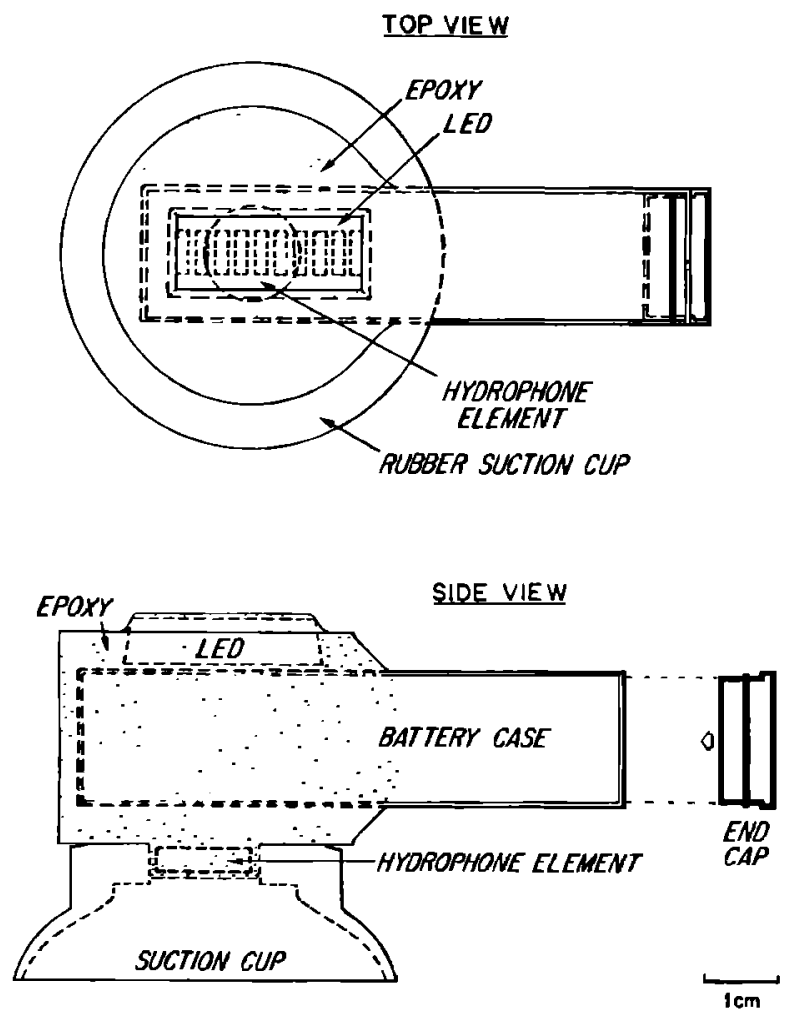

FIG. 2. Scale drawing of the vocalight. which the suction cup is attached. The battery case is sealed with an end cap machined out of acrylic plastic with a rubber O-ring. Duracell TR-177 mercury batteries $(9.8 \mathrm{~V})$ are used to power the vocalight.

Figure 3 shows a Bode plot of the frequency response of a vocalight. These measurements were made at least $2 \mathrm{~m}$ from the nearest reflecting surface in an acoustic test pool. Calibration tone bursts of 1.5 -ms duration were used to allow measurement of sound levels unaffected by echoes. Tone bursts were broadcast with a power amplifier, impedance matching transformer, and a USRD F-40 transducer. Sound levels were measured with a calibrated LC-10 hydrophone 1 $m$ from the sound source. The minimum frequency that could be tested with these short tone bursts was $2 \mathrm{kHz}$. At frequencies of greater than $25 \mathrm{kHz}$, the output of the sound projector showed amplitude modulation, presumably due to nonlinear response of the transformer at these frequencies. This determined the upper limit of calibration.

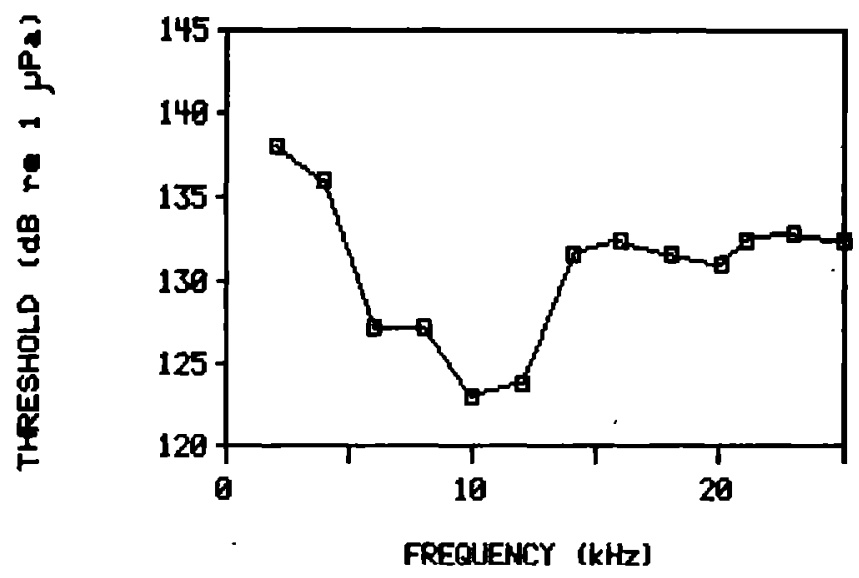

FIG. 3. Bode plot indicating the frequency response of the vocalight. Frequency response was measured in an acoustic test tank using 1.5-ms tone bursts and a calibrated hydrophone $1 \mathrm{~m}$ from the source. The threshold on the plot indicates the sound level required to light up half of the LEDs on a vocalight attached to the sound projector. 
The vocalight was attached to the flat face of the sound projector with the suction cup. This placed the vocalight hydrophone approximately $2 \mathrm{~cm}$ from the edge of the active element of the sound source and $7 \mathrm{~cm}$ from its center. At each test frequency, the sound level was adjusted to light up approximately half of the LEDs. The threshold of response of the vocalight was judged by eye. Visual estimation of this threshold level was the least precise step in the calibration, because the short duration of the tone bursts made it difficult to estimate exactly how many LEDs lit up.

\section{USE OF THE VOCALIGHT WITH DOLPHINS}

Captive bottlenosed dolphins, Tursiops truncatus, were trained to wear vocalights at Sealand, an aquarium in Brewster, MA on Cape Cod, and at the New England Aquarium in Boston. Vocalights were placed on the dolphins' melons several centimeters anterior to the blowhole. To prevent skin irritation, the suction cup on each vocalight was coated with a lubricant before use and was removed every half hour or so for repositioning. While a dolphin could shake the vocalight off by rapid sideways motion of the head, the vocalights usually remained in position during normal activities, including breaches and chases. A suitable range of amplifier gain for this device was determined by tests with the captive dolphins. The optimal amplifier gain was approximately $\mathbf{5 0}$ dB.

The vocalights were used to define the whistle repertoires of the two Tursiops held at Sealand (for a detailed description of the results of this study, see Tyack, in press). In order to determine which of the two dolphins produced a whistle, the vocalights were adjusted to have identical sensitivity. To help identify the dolphins, red LEDs were used for one vocalight, and green for the other. Dolphin sounds were picked up by a hydrophone in the pool and broadcast in air to observers around the pool, who carefully watched the vocalight nearest them. If both dolphins were nearby, one observer could sometimes follow both devices, but several observers at different locations were usually required to keep both devices under observation. When a whistle was produced, the observer called out an estimate of the number and color of the LEDs that lit up. If a whistle was heard but observers did not see any LEDs light up, they called out that none of the color of the LEDs on that device lit up. If they could not see any devices clearly, they remained silent. These comments were recorded with a microphone on a second channel of the same tape that recorded the underwater dolphin sounds.

It was seldom difficult to associate observers' comments with one whistle, unless both dolphins produced simultaneous sounds. While the loudness of the whistles varied, the 30-dB range of the vocalight was usually sufficient to identify which animal had whistled. The vocalights were adjusted to turn on several LEDs even for relatively faint whistles. Observers never reported that neither device lit up when a whistle was heard. But occasionally when very loud whistles were heard, and both dolphins were separated by only approximately $1 \mathrm{~m}$, both devices lit up fully, making it impossible to identify which animal produced the whistle.

\section{DISCUSSION}

Since the vocalight is calibrated for sound level, it may be used to estimate the source level of whistles from captive Tursiops, with several caveats. For a rough estimate, let us assume that the problems of nearfield interference effects and sound directionality would not lead to significant differences in measured levels. Figure 3 shows that for the frequency range of 5-12 kHz, sound levels of approximately $125 \mathrm{~dB}$ re: $1 \mu \mathrm{Pa}$ at $1 \mathrm{~m}$ from the sound projector were required to light up half of the LEDs. Since there is a 15-dB increase in threshold from lighting up half of the LEDs to lighting all of them, the threshold for lighting all LEDs would be approximately $140 \mathrm{~dB}$. No whistles audible to us were so faint that no LEDs lit up, which would correspond to a threshold of approximately $110 \mathrm{~dB}$. Almost all of the whistles lit up at least half of the LEDs, corresponding to a threshold of $125 \mathrm{~dB}$. Many whistles saturated the device, lighting up all LEDs. These whistles had levels of at least 140 $\mathrm{dB}$, but the maximum levels are unknown.

There are few reports on the source levels of Tursiops whistles. Fish and Turl (1976) measured the maximum levels at different frequencies of a sample of whistles from a group of approximately ten wild Tursiops. Maximum levels were measured by using a peak hold mode on a real-time spectrum analyzer. These levels ranged from $150-173 \mathrm{~dB}$ re: $1 \mu \mathrm{Pa}$ at 1 $\mathrm{m}$, which is consistent with the result reported in this paper that whistles exceeded levels of $140 \mathrm{~dB}$. Use of the vocalight shows that Tursiops can modify the levels of their whistles over a large dynamic range. This point is also made by Watkins and Schevill (1974), who measured source levels of whistles from wild spinner dolphins, Stenella longirostris, varying from 109-125 $\mathrm{dB}$ re: $1 \mu \mathrm{Pa}$ at $1 \mathrm{~m}$.

While the vocalight has only been used with captive dolphins, it might be used with wild dolphins in an application where observers could stay near a few individuals and where water visibility was good. The most serious problem would be attachment of the device. Attachment would be simplest if one could catch and restrain a dolphin, attach the device by hand, and then free the animal. It might also be possible to attach the device to dolphins near a boat, using poles or a small projectile like the suction cup-tipped arrows used by Goodyear (1981) to attach suction cups to baleen whales.

\section{ACKNOWLEDGMENTS}

Thanks to Ronald Larkin, Francis Carey, Frederick Hess, William Watkins, G. Richard Harbison, and Richard Koehler for advice, engineering assistance, and loans of equipment and space during development of the vocalight. Testing of the vocalight was performed at the New England Aquarium and at Sealand, an aquarium in Brewster, MA. Thanks to George King, the director of Sealand, Dick Gage, Janet Hester, Jack Pearson, John Prescott, Susan Sinclair, and Keith Wilson. Thanks to Charles I. Malme of Bolt Beranek and Newman Inc. for help in calibrating the vocalight. Thanks to Francis Carey, Karen Moore, William Schevill, and William Watkins for reviewing the manuscript. This research was performed with financial assistance from a 
W. H. O. I. Postdoctoral Scholar Award and N. I. H. Postdoctoral Fellowship 5-F32-NS07206. This is contribution number 5941 from the Woods Hole Oceanographic Institution.

Burdin, V. I., Reznik, A. M., Skornyakov, V. M., and Chupakov, A. G. (1975). "Communication signals of the Black Sea bottlenosed dolphin," Sov. Phys. Acoust. 20, 314-318.

Evans, W. E., and Sutherland, W. W. (1963). "Potential for telemetry in studies of aquatic animal communication," in Bio-telemetry, edited by $\mathrm{L}$. E. Slater (Pergamon, Oxford), pp. 217-224.

Fish, J. F., and Turl, C. W. (1976). "Acoustic source levels of four species of small whales," Naval Undersea Center Report Number NUC TP 547 (14 pp.).

Gish, S. L. (1979). "A quantitative description of two-way acoustic communication between captive Atlantic bottlenosed dolphins (Tursiops truncatus Montagu)," Ph.D. thesis, University of California at Santa Cruz, available from University Microfilms, Ann Arbor, MI.

Goodyear, J. (1981). "Remora tag effects the first radio tracking of an Atlantic humpback," in Fourth Biennial Conference on the Biology of Marine Mammals, Abstracts, p. 46.

Lang, T. G., and Smith, H. A. P. (1965). "Communication between dolphins in separate tanks by way of an electronic acoustic link," Science 150, 1839-1844.

Mackay, R. S. (1968). Bio-medical Telemetry (Wiley, New York), p. 359.

Popper, A. N. (1980). "Sound emission and detection by delphinids," in Cetacean Behavior: Mechanisms and Functions, edited by L. M. Herman (Wiley, New York), Chap. 1, pp. 1-52.

Tyack, P. (in press). "Whistle repertoires of two bottlenosed dolphins, Tursiops truncatus: Mimicry of signature whistles?," submitted to Behav. Ecol. Sociobiol.

Watkins, W. A., and Schevill, W. E. (1974). "Listening to Hawaiian spinner porpoises, Stenella cf. longirostris, with a three-dimensional hydrophone array," J. Mammal. 55, 319-328.

\title{
Level effects in complex stimulus discrimination
}

\author{
Christopher W. Turner and Anthony T. Cacace \\ Communication Sciences and Disorders, Syracuse University, 805 South Crouse Avenue, Syracuse, New York \\ 13210
}

(Received 23 July 1984; accepted for publication 23 July 1985)

\begin{abstract}
The dependence of spectral-shape discriminations upon presentation level was studied using stimuli consisting of a bandpass noise and a less intense pure tone. The level of the noise band was held constant $(+10 \mathrm{~dB})$ relative to the level of the tone, and the minimum change in the intensity of the tone was measured across a range of presentation levels. The results demonstrated that, for tonal frequencies located near the upper edge of the noise band, subject's optimal performance occurred at intermediate presentation levels and became considerably poorer at high levels. This result is in accordance with upward spread of masking and emphasizes that presentation level is an important parameter to consider when measuring discriminations of complex spectral shapes.
\end{abstract}

PACS numbers: 43.66.Fe, 43.66.Dc

\section{INTRODUCTION}

The ability of subjects to discriminate between complex spectral shapes has commonly been studied by determining the difference limen for an incremental change in the stimulus. Using such tasks, Turner and Van Tasell (1984) and Mason et al. (1984) noted that subject's performance was decreased at high presentation levels in tasks requiring the discrimination of small changes in the level within specific frequency regions of a complex stimulus. In a study using speechlike stimuli, Danaher et al. (1973) found that discrimination of frequency changes of a second-formant component was in some cases reduced when a first formant was added to the stimulus, particularly at high presentation levels. They suggested that intercomponent masking may affect the discrimination of spectral shapes. These previous studies demonstrated that performance in the discrimination of a given complex spectral shape may be dependent upon presentation level. This dependence would be expected to follow from the frequency pattern seen in spread of masking studies, or in other terms, the broadening of auditory excitation patterns with increasing level.

The present report describes the effect upon discrimination of a single stimulus component resulting from intercomponent masking, as a function of presentation level. The difference limen for intensity of a single pure tone was measured both for the tone in isolation and in the presence of a higher-intensity narrow-band masking noise. The frequency distance between tone and noise was varied across conditions in order to determine the effects of intercomponent masking as a function of frequency and level of the stimulus components.

\section{METHODS}

A two-interval, two-alternative forced choice task (2AFC) was used to measure discrimination performance. The subject's task was to indicate, via response buttons, the 